\title{
Reproductive Phenology of Tea (Camellia sinensis (L.) O. Kuntze) Cultivars in Sri Lanka
}

\author{
J.H.N. Piyasundara ${ }^{*}$, I.P. Wickramasinghe ${ }^{1}$, M.T.K. Gunesekara ${ }^{2}$, M.A. Wijeratne ${ }^{3}$, \\ S.A.C.N. Perera ${ }^{1}$, M.A.B. Ranathunga and A.K. Mudalige
}

\author{
Plant Breeding Division \\ Tea Research Institute of Sri Lanka \\ Sri Lanka
}

\begin{abstract}
Reproductive phenological attributes of the tea (Camellia sinensis (L.) $O$. Kuntze) plant have not been paid adequate attention until recently as the plant is being maintained continuously in the vegetative phase for commercial harvesting. However, with the introduction of improved seeds as an alternative planting material to adapt climate change scenarios and with recent advances of controlled hybridization, extensive studies on those aspects become a necessity. Therefore, the present study was conducted to assess the reproductive phenology of ten selected parental cultivars from a representative sample of three tea seed gardens; Salawa, Rembukkanda and Reucastle. Reproductive phenophases were visually rated on a non-parametric scale (0-5) monthly, for a period of 3 consecutive years. Ratings were subsequently converted into an index which represented both synchrony and intensity of phenophases. Study identified the periodicity, synchrony and intensity of reproductive phenophases in relation to the rainfall pattern of the area. Major flowering period was from September to December and major fruiting period was from April to August. The values of indices reflected significant variations of the phenological behavior among the different parental cultivars. The profuse fruit setters and profuse flower setters among the parental cultivars have been identified through the study. The information would be utilized as a base material in selecting suitable parental combinations for future gardens to ensure optimum productivity. Based on the overall results, phenological calendar and phenograms were constructed to facilitate the garden management and seed collection. The information generated could be utilized in controlled hybridization programmes to select candidate parents and to decide the timelines.
\end{abstract}

Keywords: Tea cultivars, phenology, seed gardens, phenograms, phenological calendar

\section{INTRODUCTION}

Tea (Camellia sinensis L.) is a predominantly out crossing woody perennial. Unlike in most perennials, vegetative parts of tea plant; two tender leaves and the bud are often commercially exploited. Ultimately, tea was brewed into a beverage which is known to be the most favored beverage in the world (Amarakoon, 2004). Therefore, the plant is

\footnotetext{
Department of Agricultural Biology, Faculty of Agriculture, University of Peradeniya, Sri Lanka

Ministry of Primary Industries, Colombo, Sri Lanka

3 Agronomy Division, Tea Research Institute of Sri Lanka, Thalawakale, Sri Lanka

* Corresponding author: noelpiyasundara@gmail.com
} 
commercially maintained in a vegetative phase continuously. The vegetative phenology of tea has been studied extensively due to its commercial value. However, the reproductive phenology of tea has not been paid much attention. A few studies have been reported on tea reproductive biology/floral biology (Barua, 1970; Neog and Singh, 2003; Ariyarathna et al., 2011), phenology (Barua, 1970; Bezbaruah, 1975; Ariyarathna et al., 2011), male gamatophytic competition (Muoki et al., 2007) and insect pollination (Wickramaratne and Vitarana, 1985).

Recently, improved seed materials were developed via seed gardens which have been introduced as an adaptive measure to face to effects of climate change (Piyasundara et al., 2012). Moreover, recent advances in controlled hybridization programmes are needed to be facilitated with information on reproductive phonological aspects. Therefore, extensive studies on reproductive phenology of tea cultivars are necessary. In Sri Lanka, combinations of commercial tea cultivars (clones) selected based on their known desired traits (high yield, tolerance to pest and diseases and drought) were included in tea seed gardens as parental cultivars. This was aimed to obtain "clonal seeds" via natural hybridization providing equal chance to cultivars to cross pollinate with each other. This was achieved by planting the cultivars in a specific planting arrangement. There were two types; bi-clonal gardens with only two known cultivars and poly-clonal gardens with 5-7 known cultivars (Gunasekara, 2008).

Majority of these gardens have been established in the agro-ecological region (AER) of Low Country Wet Zone 1a (WL1a) in Rubber plantations owing to the necessity of isolating them from nearest tea fields to avoid possible pollen contamination (Wickramaratne, 1981). In the gardens, tea plants are allowed to grow freely up to a few meters to encourage reproductive growth to exploit maximum seed production. Tea seed gardens provide a perfect environment to study the reproductive phenology of respective parental cultivars owing to the isolated nature, specific planting arrangement, maintenance status and known cultivar combinations. The present study was undertaken to assess reproductive phenology of some selected parental cultivars of the tea seed gardens. Mainly aiming to utilize the information to streamline the garden management, to plan productivity enhancement programmes of the gardens and to facilitate the controlled hybridization programmes.

\section{MATERIALS AND METHODS}

\section{Locations and materials}

The assessment was conducted in a representative sample of three isolated tea seed gardens. The selection was made on the basis of adequate representation of parental cultivars and the performances of the seed stocks assessed previously in the adaptive trials. All parental cultivars of the three gardens (Table 1) were included in the assessment. These cultivars have been included in recent controlled hybridizations progrmmes as well (unpublished plant breeding records, Tea Research Institute, Sri Lanka). 
Table 1. Details of the study locations (Tea seed garden)

\begin{tabular}{|c|c|c|c|c|}
\hline Status & Location & AER & $\begin{array}{l}\text { Elevation } \\
\text { (Approximately } \\
\text { m amsl) }\end{array}$ & Parental Cultivars \\
\hline $\begin{array}{l}\text { Bi- } \\
\text { Clonal }\end{array}$ & $\begin{array}{l}\text { Reucastle Estate, } \\
\text { Dehiowita, } \\
\text { Kegalle District }\end{array}$ & WL1a & 200 & TRI 3063 and S 106 \\
\hline $\begin{array}{l}\text { Poly- } \\
\text { Clonal }\end{array}$ & $\begin{array}{l}\text { Salawa Estate, } \\
\text { Hanwella, } \\
\text { Colombo District }\end{array}$ & WL1a & 25 & $\begin{array}{l}\text { TRI } 2016, \text { TRI } 2027, \\
\text { TRI } 3047, \text { TRI } 3055, \\
\text { KEN } 16 / 3 \text { and S } 106\end{array}$ \\
\hline $\begin{array}{l}\text { Poly- } \\
\text { Clonal }\end{array}$ & $\begin{array}{l}\text { Rambukkanda } \\
\text { Estate, Ratnapura, } \\
\text { Ratnapura District }\end{array}$ & WL1a & 100 & $\begin{array}{l}\text { TRI 2022, TRI 2025, } \\
\text { TRI 2027, TRI 3047, } \\
\text { TRI } 3055, \text { KEN } 16 / 3 \\
\text { and KP } 204\end{array}$ \\
\hline
\end{tabular}

\section{Visual observations/estimations on reproductive phenology}

Four main phenophases (phenological events or stages) were considered via immature flower buds, mature flower buds, open flowers, immature fruits and mature fruits. Visual observations/estimations were made on the each phenophase separately by using a nonparametric scale of 0-5 (Table 2). Constriction of the scale and the differentiation of phenophases were based on Ariyarathna et al. (2011) and field experience of authors. Visual observations were made monthly in five (5) randomly selected healthy trees per each parental cultivar in each garden for three (3) years continuously (from January 2014 to December 2016).

Table 2. Details of the non-parametric scale for visual observations/estimations

\begin{tabular}{lll}
\hline Rating & Description & Status \\
\hline 0 & Almost no abundance & Nil or very few \\
1 & Abundance is about or below 5\% of the tree & Few \\
2 & Abundance is about or below 10\% of the tree & In between medium and few \\
3 & Abundance is about or below 25\% of the tree & Medium \\
4 & Abundance is about 25-50\% of the tree & In between medium and high \\
5 & Abundance is over 50\% of the tree & High \\
\hline
\end{tabular}

\section{Quantification of phenophases}

The visual ratings were converted into an index for each phenophase by using the following formula:

$\frac{\sum \mathrm{R}}{\left(\mathrm{R}_{\mathrm{H}} \mathrm{XN}\right)}$

$\sum \mathbf{R}=$ Summation of the visual ratings given for the given phenophase (eg. flowering) for a given cultivar at a given location in a given period of time.

$\mathbf{R}_{H}=$ Highest rating of the visual scale (the value of the $R_{H}=5$ in the present study).

$\mathbf{N}=$ Total No. of individuals rated (total no. of observations). 
Monthly values of the indices were calculated initially, and annual/tri-annual averages were calculated subsequently. The index was simple ranging from $0-1$, and represents the synchrony as well as the intensity. The indices were calculated in relation to the highest rating of the visual scale $\left(\mathrm{R}_{\mathrm{H}}\right)$. Therefore, the indices could be used to compare the cultivars within each location as well as in between locations with a reasonable degree of accuracy, especially under same agro-ecological condition. The indices for five phenophases were abbreviated as; Immature Flower Bud Index (IFBI), Mature Flower Bud Index (MFBI), Open Flower Index (OFI), Immature Fruit Index (IFI) and Mature Fruit Index (MFI)

\section{Rainfall data}

According to the results made by Muoki et al. (2007) in Kenya and Ariyarathna et al. (2011) in Sri Lanka and the authors' field experiences, flowering of tea has close synchrony with the rain fall pattern of the respective area. Therefore, rain fall data from 2013-2015 of three (3) locations were obtained to supplement the phenological observations.

\section{Data analyses}

Average linkage cluster analysis was performed to group the parental cultivars based on their flowering and fruit setting levels using Minitab (2014) software.

\section{RESULTS AND DISCUSSION}

\section{Phenology of flower buds}

Immature flower buds were observed in different intensities throughout the year during three years of study period, irrespective to the cultivar or location. The intensity was enhanced from January to March and comparatively reduced from April to June (Figure 1(a)). The situation was similar in the case of mature flower buds (Figure 1(b)), but reduced intensities were corresponded to immature flower buds.

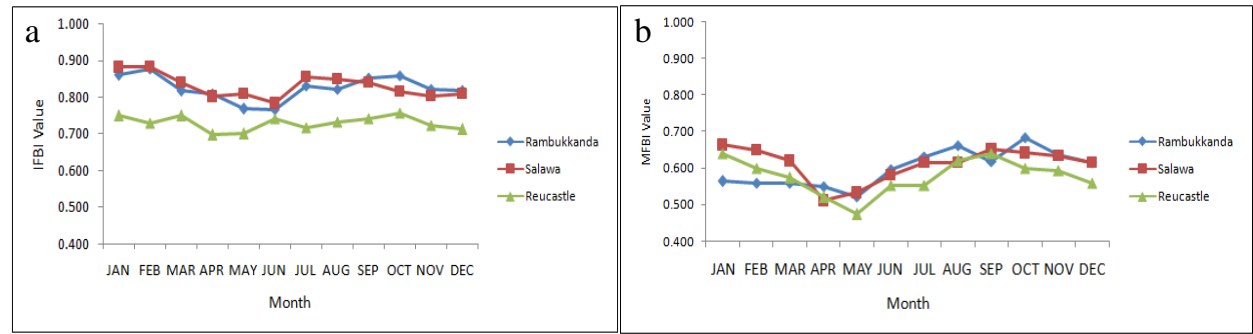

\section{Figure 1. Monthly tri-annual means of IFBI (a) and MFBI (b) of tea}

Although the flower bud patterns were basically similar in 3 locations, considerable variations were observed in intensity among the parental cultivars (Table 3). Among the parental cultivars, TRI 2027 exhibited the highest intensity of immature flower buds at both Rambukkanda and Salawa. However, the higher immature flower bud intensities were observed in TRI 2027 and as well as in TRI 3047. This was not reflected in corresponding mature flower bud intensity levels (Table 3). This reduction could be partly attributed to the notable level of drying of flower buds observed in these two cultivars. 
Table 3. Calculated annual and tri-annual means of immature flower bud index (IFBI) and mature flower bud index (MFBI) of tea in 3 study locations

\begin{tabular}{|c|c|c|c|c|c|c|c|c|}
\hline \multicolumn{9}{|c|}{ Rambukkanda Poly-Clonal Tea Seed Garden } \\
\hline & \multicolumn{4}{|c|}{ IFBI } & \multicolumn{4}{|c|}{ MFBI } \\
\hline & Y1 & Y2 & Y3 & 3YM & Y1 & Y2 & Y3 & 3YM \\
\hline TRI 2022 & 0.813 & 0.857 & 0.870 & 0.847 & 0.673 & 0.643 & 0.560 & 0.626 \\
\hline TRI 2025 & 0.837 & 0.813 & 0.847 & 0.832 & 0.707 & 0.640 & 0.617 & 0.654 \\
\hline TRI 2027 & 0.947 & 0.957 & 0.913 & 0.939 & 0.687 & 0.603 & 0.513 & 0.601 \\
\hline TRI 3047 & 0.823 & 0.797 & 0.820 & 0.813 & 0.593 & 0.507 & 0.530 & 0.543 \\
\hline TRI 3055 & 0.877 & 0.833 & 0.873 & 0.861 & 0.730 & 0.657 & 0.587 & 0.658 \\
\hline KEN $16 / 3$ & 0.777 & 0.727 & 0.820 & 0.774 & 0.637 & 0.583 & 0.573 & 0.598 \\
\hline KP 204 & 0.743 & 0.670 & 0.727 & 0.713 & 0.573 & 0.533 & 0.450 & 0.519 \\
\hline Garden & 0.831 & 0.808 & 0.839 & 0.826 & 0.657 & 0.595 & 0.547 & 0.600 \\
\hline \multicolumn{9}{|c|}{ Salawa Poly-Clonal Tea Seed Garden } \\
\hline TRI 2016 & 0.810 & 0.840 & 0.827 & 0.826 & 0.627 & 0.637 & 0.590 & 0.618 \\
\hline TRI 2027 & 0.987 & 0.933 & 0.953 & 0.958 & 0.667 & 0.623 & 0.560 & 0.617 \\
\hline TRI 3047 & 0.793 & 0.793 & 0.817 & 0.801 & 0.597 & 0.527 & 0.533 & 0.552 \\
\hline TRI 3055 & 0.890 & 0.870 & 0.853 & 0.871 & 0.740 & 0.740 & 0.620 & 0.700 \\
\hline KEN $16 / 3$ & 0.780 & 0.753 & 0.810 & 0.781 & 0.597 & 0.587 & 0.593 & 0.592 \\
\hline S 106 & 0.740 & 0.717 & 0.797 & 0.751 & 0.607 & 0.630 & 0.527 & 0.588 \\
\hline Garden & 0.833 & 0.818 & 0.843 & 0.831 & 0.639 & 0.624 & 0.571 & 0.611 \\
\hline \multicolumn{9}{|c|}{ Reucastle Bi- Clonal Tea Seed Garden } \\
\hline TRI 3063 & 0.745 & 0.660 & 0.733 & 0.713 & 0.617 & 0.580 & 0.427 & 0.541 \\
\hline S 106 & 0.810 & 0.687 & 0.743 & 0.747 & 0.670 & 0.633 & 0.537 & 0.613 \\
\hline Garden & 0.778 & 0.673 & 0.738 & 0.730 & 0.644 & 0.607 & 0.482 & 0.578 \\
\hline
\end{tabular}

\section{Phenology of flowering}

Similar to flower buds, flowering were observed in different intensities throughout the year, with well demarcated peaks or seasonal variations (Figure 2). The phenomenon has been previously described as common in the near equator regions (Barua, 1989).Two peaks could be observed in flowering pattern in all three locations (Figure 2), an extended peak from September to December and a brief peak in July. The former was identified as the major flowering peak and the later as the off- peak.

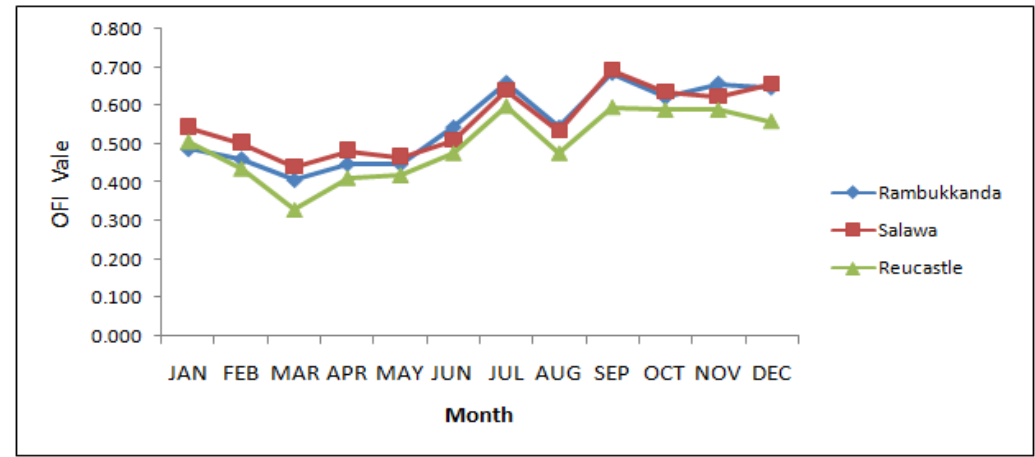

Figure 2. Monthly tri-annual means of OFI of tea 
The periodicity of the peaks were deviated from the previous reports by Ariyarathna et al., 2011 at the tea germplasm in Up country (AER-WU1), Sri Lanka where major flowering peak was observed from February to March and two off-peaks were in July and November. The deviations may be attributed to different agro-ecolgical condition as well as to specific cultivar combination and planting arrangement of the seed gardens and germplasm. Although the flowering pattern is basically similar in all three locations, considerable variations in flowering intensties were observed among the parental cultivars (Table 4). Moreover, the similar OFI values were obtained in same order across the locations when the same cultivar was included in two locations, demonstrating the specific potential of the cultivar under same agro-ecological condition. This scenario confirmed the validity of the index across locations in same agro-ecological region.

The resulting dendrogrames of average linkage cluster procedure of the OFI values Salawa (Figure 3(a)) and Rambukkanda (Figure 3(b)) clearly delineate the cultivars into discreet groups based on the intensity of flowering. Based on the results of flowering phenology assessment the parental cultivars were categorized into two discreet groups: profuse flower setters; TRI 2016, TRI 2022, TRI 2025, TRI 3055, KEN 16/3, S 106 and moderate flower setters; TRI 2027, TRI 3047, TRI 3063, KP 204.

Table 4. Calculated annual and tri-annual means of open flower index (OFI) of the tea

\begin{tabular}{|c|c|c|c|c|}
\hline \multicolumn{5}{|c|}{ Rambukkanda Poly-Clonal Tea Seed Garden } \\
\hline & Y1 & $\mathbf{Y 2}$ & Y3 & 3YM \\
\hline TRI 2022 & 0.643 & 0.617 & 0.543 & 0.601 \\
\hline TRI 2025 & 0.677 & 0.610 & 0.540 & 0.609 \\
\hline TRI 2027 & 0.553 & 0.543 & 0.433 & 0.510 \\
\hline TRI 3047 & 0.503 & 0.463 & 0.393 & 0.453 \\
\hline TRI 3055 & 0.680 & 0.617 & 0.563 & 0.620 \\
\hline KEN 16/3 & 0.647 & 0.587 & 0.523 & 0.586 \\
\hline KP 204 & 0.507 & 0.493 & 0.413 & 0.471 \\
\hline Garden & 0.601 & 0.561 & 0.487 & 0.550 \\
\hline \multicolumn{5}{|c|}{ Salawa Poly-Clonal Tea Seed Garden } \\
\hline & Y1 & $\mathbf{Y} 2$ & Y3 & 3YM \\
\hline TRI 2016 & 0.583 & 0.593 & 0.553 & 0.577 \\
\hline TRI 2027 & 0.553 & 0.520 & 0.427 & 0.500 \\
\hline TRI 3047 & 0.510 & 0.510 & 0.440 & 0.487 \\
\hline TRI 3055 & 0.670 & 0.653 & 0.593 & 0.639 \\
\hline KEN 16/3 & 0.630 & 0.573 & 0.583 & 0.596 \\
\hline S 106 & 0.603 & 0.600 & 0.503 & 0.569 \\
\hline Garden & 0.592 & 0.575 & 0.517 & 0.561 \\
\hline \multicolumn{5}{|c|}{ Reucastle Bi -Clonal Tea Seed Garden } \\
\hline & Y1 & Y2 & Y3 & 3YM \\
\hline TRI 3063 & 0.522 & 0.513 & 0.333 & 0.456 \\
\hline S 106 & 0.588 & 0.565 & 0.477 & 0.543 \\
\hline Garden & 0.555 & 0.539 & 0.405 & 0.500 \\
\hline
\end{tabular}

OFI= Open Flower Index; Y= Year; $3 Y M=$ Tri-annual Mean 


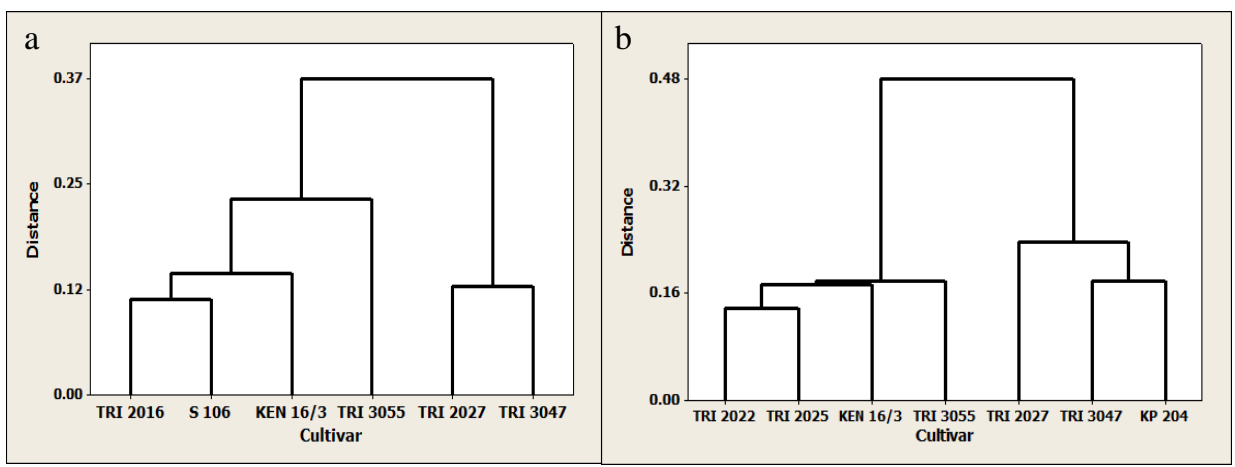

Figure 3. Dendrogrames obtained by average linkage cluster analysis of the OFI values of Salawa (a) and Rambukkanda (b), demonstrating the grouping of cultivars based on their flowering levels

\section{Phenology of fruiting}

The Table 5 represents the calculated annual and tri-annual means of immature fruit index (IFI) and mature fruit index (MFI) of the 3 experimental locations. Unlikely, in flower buds and flowers, the occurrence of fruits was more confined to a few months of the year in all three locations. Immature fruits were observed in peak from November/December and continued up to April (Figure 4(a)) and the mature fruits were observed in peak from April to August (Figure 4(b)) in all three locations. The period from April to August where mature fruits were in peak was identified as the peak fruiting period (or cropping season in agronomical terms). The cropping season coincides with the nursery season of the area (Low country). The scenario has been reported as common in the majority of the tropical crops, whereas the cropping season occurred when there is a conducive environment for seed germination (Frankie et al., 1974). As flowering to fruit set take about 8-12 months, the major flowering peak from September to December. As well as the off peak in July of the current year is responsible for the major fruit crop from April to August in next year.

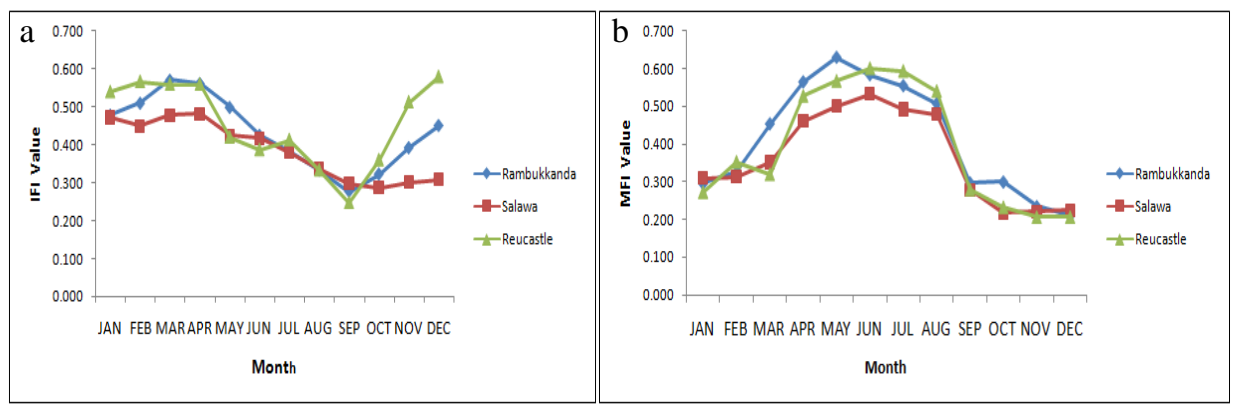

Figure 4. Monthly tri-annual means of IFI (a) and MFI (b) values of tea 
Table 5. Calculated annual and tri-annual means of immature fruit index (IFI) and mature fruit index (MFI) of tea

\begin{tabular}{|c|c|c|c|c|c|c|c|c|}
\hline \multicolumn{9}{|c|}{ Rambukkanda Poly-Clonal Tea Seed Garden } \\
\hline & \multicolumn{4}{|c|}{ IFI } & \multicolumn{4}{|c|}{ MFI } \\
\hline & Y1 & Y2 & Y3 & 3YM & Y1 & $\mathbf{Y} 2$ & Y3 & 3YM \\
\hline TRI 2022 & 0.600 & 0.633 & 0.490 & 0.574 & 0.577 & 0.610 & 0.477 & 0.554 \\
\hline TRI 2025 & 0.607 & 0.570 & 0.457 & 0.544 & 0.580 & 0.547 & 0.417 & 0.514 \\
\hline TRI 2027 & 0.477 & 0.430 & 0.373 & 0.427 & 0.470 & 0.423 & 0.365 & 0.419 \\
\hline TRI 3047 & 0.000 & 0.000 & 0.000 & 0.000 & 0.000 & 0.000 & 0.000 & 0.000 \\
\hline TRI 3055 & 0.483 & 0.453 & 0.393 & 0.443 & 0.440 & 0.413 & 0.350 & 0.401 \\
\hline KEN $16 / 3$ & 0.640 & 0.610 & 0.513 & 0.588 & 0.643 & 0.593 & 0.490 & 0.576 \\
\hline KP 204 & 0.507 & 0.473 & 0.397 & 0.459 & 0.470 & 0.410 & 0.387 & 0.422 \\
\hline Garden & 0.473 & 0.453 & 0.375 & 0.434 & 0.454 & 0.428 & 0.355 & 0.412 \\
\hline \multicolumn{9}{|c|}{ Salawa Poly-Clonal Tea Seed Garden } \\
\hline & \multicolumn{4}{|c|}{ IFI } & \multicolumn{4}{|c|}{ MFI } \\
\hline & Y1 & Y2 & Y3 & 3YM & Y1 & Y2 & Y3 & 3YM \\
\hline TRI 2016 & 0.550 & 0.557 & 0.480 & 0.529 & 0.537 & 0.479 & 0.470 & 0.495 \\
\hline TRI 2027 & 0.407 & 0.460 & 0.393 & 0.420 & 0.353 & 0.363 & 0.353 & 0.357 \\
\hline TRI 3047 & 0.000 & 0.000 & 0.000 & 0.000 & 0.000 & 0.000 & 0.000 & 0.000 \\
\hline TRI 3055 & 0.397 & 0.407 & 0.390 & 0.398 & 0.380 & 0.377 & 0.343 & 0.367 \\
\hline KEN $16 / 3$ & 0.530 & 0.550 & 0.523 & 0.534 & 0.517 & 0.567 & 0.500 & 0.528 \\
\hline S 106 & 0.453 & 0.463 & 0.393 & 0.437 & 0.497 & 0.473 & 0.363 & 0.444 \\
\hline Garden & 0.389 & 0.406 & 0.363 & 0.386 & 0.381 & 0.377 & 0.338 & 0.365 \\
\hline \multicolumn{9}{|c|}{ Reucastle Bi -Clonal Tea Seed Garden } \\
\hline & \multicolumn{4}{|c|}{ IFI } & \multicolumn{4}{|c|}{ MFI } \\
\hline & Y1 & Y2 & Y3 & 3YM & Y1 & Y2 & Y3 & 3YM \\
\hline TRI 3063 & 0.467 & 0.393 & 0.287 & 0.382 & 0.420 & 0.367 & 0.283 & 0.357 \\
\hline S 106 & 0.600 & 0.593 & 0.400 & 0.531 & 0.497 & 0.457 & 0.327 & 0.427 \\
\hline Garden & 0.533 & 0.493 & 0.343 & 0.457 & 0.458 & 0.412 & 0.305 & 0.392 \\
\hline
\end{tabular}

The resulting dendrogrames of average linkage cluster procedure of the OFI values Salawa (Figure 5(a)) and Rambukkanda (Figure 5(b)) clearly delineate the cultivars into discreet groups based on the intensity of flowering. Based on the results the cultivars could be categorized into two discreet groups such as profuse flower setters; TRI 2016, TRI 2022, TRI 2025, KEN 16/3, S 106 and moderate flower setters; TRI 2027, TRI 3047, TRI 3063, KP 204. TRI 3047 which did not set fruits at all during the study period not fit any of the above categories. 


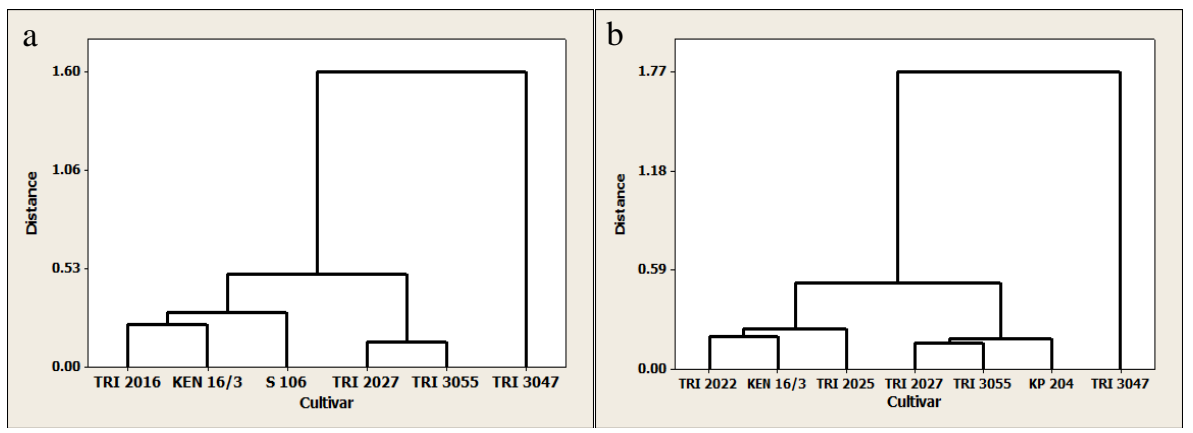

\section{Figure 5. Dendrogrames obtained by average linkage cluster analysis of the MFI values of Salawa (a) and Rambukkanda (b)}

When examine the periodicity of flowering and fruiting peaks, it was evident that irrespective of the cultivars the periodicity of flowering and fruiting peaks was basically similar in all study locations which may be attributed to the same agro-ecological condition. The difference occurred in the intensity levels, may be genetically governed and thus is cultivar specific. Both flowering and fruiting peaks are occurred in rainy season, where both phenophases occurred in comparatively reduced intensities in dry months (January to March) suggesting the close relationship between the rain fall pattern and flowering and fruit set pattern. The three locations are situated in the same agroecological region (WL1a) and the low intensities of flowering and fruiting were occurred in dry months (January to March). Whereas, peaks were occurred alternatively in wet months. Thus it was clear that there may be a considerable relationship between the patterns of phenology and rainfall.

\section{Reproductive phenology vs rainfall}

The study was conducted in agro-ecological region of WL1a. The region receives a reasonable amount of rainfall in three out of the four main rain-fall seasons in Sri Lanka, leaving out the Northeast monsoon. The 75\% expectancy of annual rainfall of the area is over $3200 \mathrm{~mm}$ (Punayawardena et al., 2003 and Punayawardena, 2008). To assess the reproductive phenology of locations in relation to the rainfall pattern, monthly rainfall records were obtained during the period of four years, commencing from the previous year before the start of the study and till the completion of the study. To demonstrate the relationship between phenological patern and rainfall pattern, monthly values OFI (Flowering) and MFI (Fruiting) of the respective location (overall value) were graphically represented with the monthly rain fall figures of the location (Figure 6(a), (b) and (c)). A hypothetical graph (Figure 6(d)) constructed based on 75\% expectancy of rainfall of the agro-ecological region WL1a, based on Punayawardena et al, (2003) and Punayawardena, (2008), was also included to demonstrate the deviations from the normal rainfall pattern.

Both flowering and fruit set is comparatively lower in the dry period of the region (January - March). However, the intensity of flower buds was comparatively higher during the study period. The moisture stress might be triggering the plant to produce more no. of flower buds, but keep the main phenophases in a dormant stage. The major fruiting season occurred from April-August, where the area receives south-west and inter-monsoonal rains. Therefore, it is evident that the fruit ripening needs an initial dry 
period as well as adequate rainfall at the time of maturity. The off peak flowering is occurred in July and the peak flowering is occurred from September to December in the rainy season, where the area receives rains from inter-monsoonal (mainly) and north-east monsoon, suggesting that flower blooming needs adequate rainfall. As flowering to fruit set take about 8-12 months, the major flowering peak from September to December as well as the off peak in July of the current year were responsible for the major fruit crop from April to August in the following year. In the first two years of study, notable variations in rainfall patterns were not evident, so as the flowering and fruit set pattern. However, a considerable decrease in flowering and fruit set was occurred and, a similar situation was reflected in rainfall pattern during $3^{\text {rd }}$ year (2016).

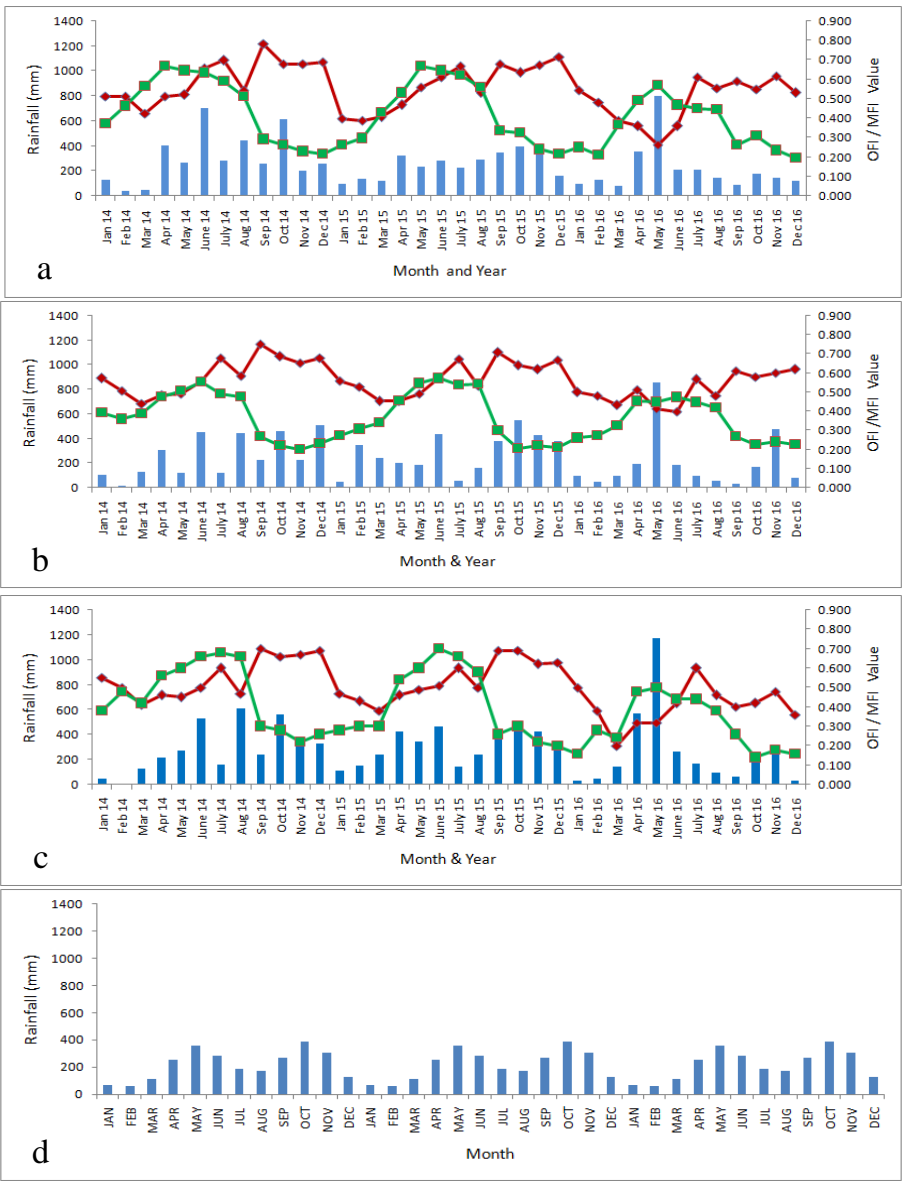

Rainfall (mm) $\longrightarrow$ - Flowering (OFI) -Fruiting (MFRI)

Figure 6. Graphical representation of monthly OFI \& MFRI values of tea vs rainfall of 3 study locations; Rambukkanda (a), Salawa (b) and Reucastle (c) for the study period of 3 consecutive years (2014-2016); hypothetical (d) graph constructed based on $75 \%$ expectancy of rainfall

The lowest total rainfall was recorded in the $3^{\text {rd }}$ year (2016) in all study locations. Moreover, a considerable variation in rainfall distribution was also observed, where all 
three locations received a very high rainfall than expected in May while considerably lower rainfalls than expected was recorded from September to December. Under normal circumstances, the area (WL1a) gets adequate rainfall from south-west monsoon and inter-monsoon rains from September to December. This facilitates the blooming of flowers as well as minimizing the drying of flowers. Hence, notable decrease in rainfall was resulted negatively by providing unconducive environment for blooming of flowers and eventually causing a decrease in flowering intensity. The falling of a certain amount of immature fruits and mature flower bud is occurred in May. Normally, this does not affect the fruit set levels notably, but the rainfall was much higher than expected and caused to notable fall of fruits as well as matured flower buds, subsequently leads to a decrease in the intensity of the open flowers and fruits in 2016. The overall results confirmed the close relationship of flowering and the rainfall pattern, which has been previously observed by Muoki et al. (2007) in Kenya and Ariyarathna et al. (2011) in Sri Lanka. Moreover, it gives evidence that not only the flowering pattern but also the fruiting pattern has close synchrony with the rainfall pattern.

\section{Implications of the findings of the study}

The profuse flower setters and fruit setters that were identified through the study can be utilized as the base material in selecting the compatible parental combinations in future gardens ensuring better seed production. The information that was generated on flowering phenology of different cultivars would be utilized in planing the future controll hybrdization programmes.Based on the flowering and fruiting periodicity and the relationship with the rainfall pattern of the area, a "Phenological Calendar" (Figure 7) was constructed as an initial step to provide the basic schedule to streamline the garden management.

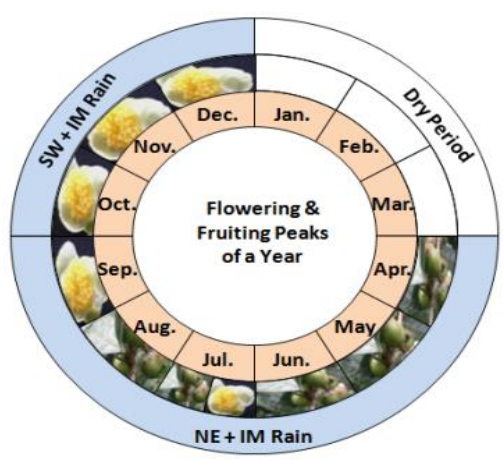

Figure 7. Phenological calendar representing flowering and fruiting peaks of tea in relations to the rainfall seasons of the study area

$N E+I M=$ North East and Intermonsoonal Rains $/ S W+I M=$ South West and Intermonsoonal Rains

Based on the information generated on flowering and fruit set levels of each tea cultivar at each location, "Phenograms" of flowering (Figure 8) and fruiting (Figure 9) were constructed. When constructing phenograms, it was considered that the flowering or fruiting was in peak. The phenograms reflected the cultivar specific as well as garden specific of flowering /fruiting peaks. Moreover, the phenograms confirm the cultivar specific variations in synchrony /intensity of flowering / fruiting. Extended and intensive 
flowering peaks were exhibited by certain cultivars (TRI 2016, TRI 2022, TRI 2025, TRI 3055 , KEN 16/3, and S 106) and shorter flowering peaks with lower intensity levels were exhibited cultivars like TRI 2027, TRI 3047, TRI 3063 and KP 204. In the case of fruiting there were cultivars with extended and intensive fruiting peaks (TRI 2016, TRI 2022, TRI 2025 and KEN 16/3), a cultivar with regular but intensive fruiting peaks (S 106), cultivars with regular fruiting peaks but comparatively reduced intensity levels (TRI 2027, TRI 3055, TRI 3063) and a cultivar with no fruit set (TRI 3047) at all.

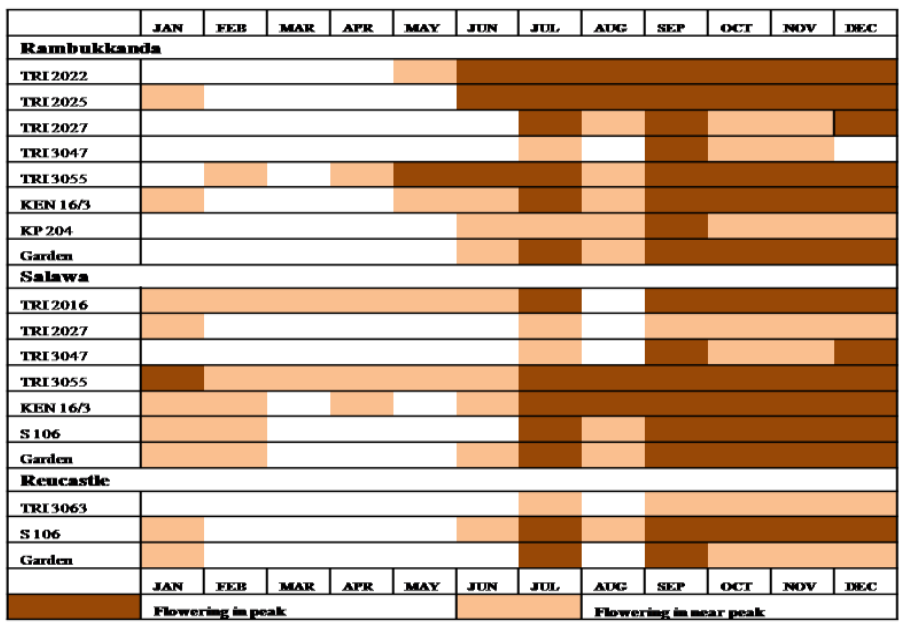

Figure 8. Flowering phenogram demonstrating the flowering peaks of tea cultivars/gardens

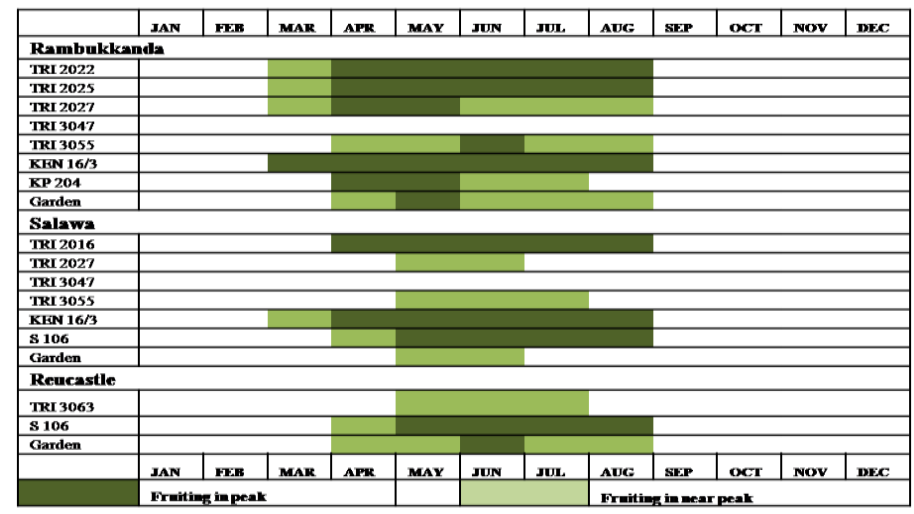

Figure 9. Fruiting phenogram demonstrating the flowering and fruiting peaks of the year of cultivars/ gardens

The "Phenological Calendar" along with phenograms will be utilized in planning seed collection, distribution programmes and nursery programmes, thereby minimizing pre harvest and post-harvest crop (seed) loss. In addition, cultural operations such as fertilizing and pest and diseases management strategies could also be planned accordingly. Fruiting phengrams provides an accurate estimation on actual productivity levels of each location. It is clearly evident that due to cumulative effect of parental cultivars, the gardens showed a low productivity levels confirming current situation of 
inadequate seed production to fulfill the grower demand. The phenograms could be utilized for the garden management in more specific manner, "Cultivar specific manner" and it also provides the basic time frame for the controlled hybridization programmes among these cultivars.

\section{CONCLUSIONS}

The peak flowering period of Tea (Camellia sinensis L.) was from September to December of the year and an off-peak in July. The main cropping period was from April to August. The results reflected considerable variations among the studied cultivars with respect to reproductive phenological attributes. The profuse fruit setters (TRI 2016, TRI 2022, TRI 2025, KEN 16/3 and S 106) and profuse flower setters (TRI 3055, TRI 2016, TRI 2022, TRI 2025, KEN $16 / 3$ and S 106) were identified through the study. A phenological calendar and the phenograms, constructed based on the overall outcome of the study would be utilized to plan seed collection programmes and other cultural practices of tea gardens.

\section{ACKNOWLEDGEMENTS}

Co-operation extended by; Managers and staff of Salawa, Rambukkanda and Reucastle Estates, in conducting the assessments and providing the rain fall data; Mr. A.M.U. Liyanage \& Mr. A.K.J. Athukorala (Plant Breeding Division, TRI) in collecting data are gratefully acknowledged. This study was funded by the Tea Research Institute of Sri Lanka.

\section{REFERENCES}

Amarakoon, T. (2004), Tea for Health, Tea Research Institute, Sri Lanka.

Ariyarathna, H.A.C.K., Gunasekare, M.T.K., Arachchi, J.D.K., Paskarathevan, R., Ranaweera, K.K., Ratnayake, M. and Kumara, J.B.D.A.P. (2011). Morpho-physiological and phenological attributes of reproductive biology of tea (Camellia sinensis (L.) O. Kuntze) in Sri Lanka. Euphytica. 181, 203-215.

Barua, P.K. (1970). Flowering habit and vegetative behavior in tea (Camellia sinensis L) seed trees in North-East India. Ann. of Bot. 34, 721-735.

Barua, D.N. (1989). Science and Practice in Tea Culture, Tea Research Association, Calcutta, Jorhat, India.

Bezbaruah, H.P. (1975). Development of flower, pollination and seed-set in tea in NorthEast India. Two and Bud. 22(1), 25-30.

Frankie, G.W., Baker, H.G. and Opler, P.A. (1974). Comparative phonological studies of trees in tropical wet and dry forests in the lowlands of Costa Rica. J. Ecol. 62, 881-913. 
Gunesekara, M.T.K. (2008). Planting materials. pp. 34-49. In; Handbook on Tea. Zoysa A.K.N. (Ed.) Tea Research Institute, Sri Lanka.

Muoki, R.C., Wachira, E.N., Pathak, R. and Kamunya, S.M. (2007). Potential male gametophyte competition among Camellia sinensis genotypes in isolated bi-clonal seed orchards. Afr. Crop Sci. J. 15(2), 59-66.

Neog, B. and Singh, I.D. (2003). Study of floral biology of some cultivated tea (Camellia spp.) growing in the upper Assam region of North East India. J. Plant Crops. 31(1), 5052 .

Piyasundara, J.H.N., Ranatunga, M.A.B., Gunasekare, M.T.K., Upali, P.D., Paskaradevan, R., KottawaArachchi, J.D., Ranaweera, K.K., Sarathchandra, T.M., and Mudalige, A.K. (2012). Improved tea seeds as a source of planting material: A strategy for adaptation to climate change. pp. 1-9. In: Hettiarachchi, L.S.K. and Abeysinghe, I.S.B. (Ed) Proceedings of the Fourth Symposium on Plantation Crop Research. Tea Research Institute, Talawakelle, Sri Lanka.

Punayawardena, B.V.R., Bandara, T.M.J., Munasinghe, M.A.K. Banda, Pushpakaumara, S.M.V. (2003). Map and accompanying text of agro ecological regions of Sri Lanka Natural Resources Management Centre, Department of Agriculture, Sri Lanka.

Punayawardena, B.V.R. (2008). Rainfall and Agro-ecological regions of Sri Lanka (Sinhala). Department of Agriculture, Sri Lanka.

Wickramarathne, M.R.T. (1981). Tea clones and seeds - an appraisal. Tea Bull. 1(2), 720.

Wickramaratne, M.R.T. and Vitarana, S.I. (1985). Insect pollination of tea (Camellia sinensis L.) in Sri Lanka. Tropical Agriculture (Trinidad and Tobago), 62(3), 243-247. 